\title{
A QUASI-LINEAR EVOLUTION EQUATION AND THE METHOD OF GALERKIN
}

\author{
R. W. DICKEY ${ }^{1}$
}

\begin{abstract}
In this paper it is shown that under specified conditions on the initial data a certain infinite coupled system of ordinary differential equations has a solution satisfying an auxiliary convergence condition. The infinite system discussed is essentially the Galerkin expansion of the solution to a given quasi-linear wave equation. The results obtained suffice to prove the existence of a solution to this wave equation.
\end{abstract}

1. Introduction. The purpose of this paper is to prove the existence of solutions to the infinite system of ordinary differential equations

$$
\begin{aligned}
\ddot{T}_{j}+C_{0} j^{2} T_{j}+C_{1} j \int_{0}^{\pi}\left(\sum_{l=1}^{\infty} l T_{l} \cos l x\right)^{3} \cos j x d x=0, & \\
& j=1,2, \cdots, \infty
\end{aligned}
$$

$\left(C_{0}>0, C_{1} \geqq 0\right)$ which satisfy the initial data

$$
\begin{aligned}
& T_{j}(0)=\alpha_{j}, \\
& \dot{T}_{j}(0)=\beta_{j},
\end{aligned}
$$

and the auxiliary condition

$$
\sum_{j=1}^{\infty} j^{6} T_{j}^{2}<\infty
$$

The infinite system (1.1) is related to the quasi-linear 'string' equation

$$
w_{t t}-\left(a_{0}+a_{1} w_{x}^{2}\right) w_{x x}=0 \quad\left(a_{0}>0, a_{1} \geqq 0\right) .
$$

In fact it may be shown that proving the existence of a solution to (1.1) satisfying (1.2) and (1.3) is sufficient to prove the existence of a classical

Received by the editors March 13, 1972.

AMS (MOS) subject classifications (1970). Primary 34A35; Secondary 35L60.

${ }^{1}$ This research was supported by the National Science Foundation, Contract No. GP-28699 and the Wisconsin Alumni Research Foundation while the author was an S.R.C. Senior Visiting Fellow at the University of Newcastle upon Tyne, Newcastle upon Tyne, England.

(c) American Mathematical Society 1973 
solution to (1.4) satisfying the initial data

$$
\begin{aligned}
& w(x, 0)=f(x)=\sum_{j=1}^{\infty} \alpha_{j} \sin j x, \\
& w_{t}(x, 0)=g(x)=\sum_{j=1}^{\infty} \beta_{j} \sin j x,
\end{aligned}
$$

and boundary data

$$
w(0, t)=w(\pi, t)=0 .
$$

The actual details are discussed at the end of $\S 2$. For present purposes it suffices to note that if equation (1.4) has a sufficiently differentiable solution satisfying the boundary conditions (1.6), this solution can be written in the form

$$
w(x, t)=\sum_{j=1}^{\infty} T_{j}(t) \sin j x .
$$

The system of equations (1.1) results upon formally substituting this Fourier series into (1.4), multiplying by $\sin j x$, and integrating the resulting expression from 0 to $\pi$. Related results for the more elementary Föppl string equation [1] and Von Karman beam equation [2] have been discussed in [3], [4], and [5]. Existence and nonexistence of solutions to equations of the form (1.4) has been treated in [7], [8], and [9].

2. Existence. In order to prove the existence of solutions to (1.1) it is convenient to begin by discussing related finite systems of equations. In particular define functions $T_{j . v}$ to be solutions of

$$
\ddot{T}_{j, N}+C_{0} j^{2} T_{j, N}+C_{1} j \int_{0}^{\pi}\left(\sum_{l=1}^{N} l T_{l, N} \cos l x\right)^{3} \cos j x d x=0
$$

satisfying (1.2) for $j=1,2, \cdots, N$ and $T_{j . N} \equiv 0$ for $j>N$. It is of course necessary to show that (2.1) actually has a solution. However, this is easily done since (2.1) has associated with it a Lipschitz constant (depending on $N)$. Thus the method of successive approximation may be used to prove the existence of a solution locally (cf. [6]) and the continuation of the solution for all $t \geqq 0$ is guaranteed by the fact that (2.1) is a Hamiltonian system, i.e. solutions of (2.1) satisfy the energy identity. where

$$
\sum_{j=1}^{N} \dot{T}_{j . N}^{2}+C_{0} \sum_{j=1}^{N} j^{2} T_{j . N}^{2}+\frac{C_{1}}{2} \int_{0}^{\pi}\left(\sum_{j=1}^{N} j T_{j, N} \cos j x\right)^{4} d x=h_{N}
$$

$$
h_{N}=\sum_{j=1}^{N} \beta_{j}^{2}+C_{0} \sum_{j=1}^{N} j^{2} \alpha_{j}^{2}+\frac{C_{1}}{2} \int_{0}^{\pi}\left(\sum_{l=1}^{N} l x_{l} \cos l x\right)^{4} d x .
$$

The above procedure cannot be applied directly to (1.1) since, due to the nature of the nonlinear term as $j \rightarrow \infty,(1.1)$ is not Lipschitz continuous. 
Thus the object will be to show that solutions of (2.1) converge to a solution of (1.1) as $N \rightarrow \infty$.

If $h_{N}$ converges as $N \rightarrow \infty$, i.e. if

$$
\lim _{N \rightarrow \infty} h_{N}=h<\infty,
$$

it follows from (2.2) that $\left|T_{j, N}\right|$ and $\left|\dot{T}_{j, N}\right|$ are uniformly bounded independent of $N$. Consequently the Arzela-Ascoli lemma (cf. [6]) implies that on any closed subinterval $0 \leqq t \leqq t^{*}<\infty$ ( $t^{*}$ fixed but arbitrarily large) there is a subsequence $\left\{T_{j, N_{i}}\right\}$ which converges uniformly to a continuous function $T_{j}$ on the interval $0 \leqq t \leqq t^{*}$. In order to prove that the functions $T_{j}$ are solutions of (1.1) it is necessary to obtain a better estimate on the functions $T_{j, N}$ than that furnished by the energy estimate (2.2).

The necessary estimate follows upon multiplying $(2.1)$ by $j^{4} \dot{T}_{j, N}$ and summing over $j$. The resulting expression may be written

$$
\frac{1}{2} \frac{d}{d t}\left(\sum_{j=1}^{N} j^{4} \dot{T}_{j, N}^{2}+C_{0} \sum_{j=1}^{\infty} j^{6} T_{j, N}^{2}\right)+C_{1}\left\langle\left(w_{x}^{(N)}\right)^{3}, w_{x x x x x t}^{(N)}\right\rangle=0
$$

where

and

$$
w^{(N)}=\sum_{j=1}^{N} T_{j, N} \sin j x
$$

$$
\langle u, v\rangle=\int_{0}^{\pi} u(x) v(x) d x .
$$

After two integrations by parts it is found that

$$
\left\langle\left(w_{x}^{(N)}\right)^{3}, w_{x x x x x t}^{(N)}\right\rangle=6\left\langle w_{x}^{(N)} w_{x x}^{(N)^{2}}, w_{x x x t}^{(N)}\right\rangle+3\left\langle w_{x}^{(N)^{2}} w_{x x x}^{(N)}, w_{x x x t}^{(N)}\right\rangle
$$

or

$$
\begin{aligned}
\left\langle w_{x}^{(N)^{3}}, w_{x x x x x t}^{(N)}\right\rangle= & \frac{3}{2}(d / d t)\left\langle w_{x}^{(N)^{2}}, w_{x x x}^{(N)^{2}}\right\rangle \\
& -3\left\langle w_{x}^{(N)} w_{x t}^{(N)}, w_{x x x}^{(N)^{2}}\right\rangle+6\left\langle w_{x}^{(N)} w_{x x}^{(N)^{2}}, w_{x x x t}^{(N)}\right\rangle .
\end{aligned}
$$

Equations (2.5) and (2.9) imply that solutions of (2.1) satisfy the identity

$$
(d / d t) E_{N}=6\left\langle w_{x}^{(N)} w_{x t}^{(N)}, w_{x x x}^{(N)^{2}}\right\rangle-12\left\langle w_{x}^{(N)} w_{x x}^{(N)^{2}}, w_{x x x t}^{(N)}\right\rangle
$$

where

$$
\begin{aligned}
E_{N}= & \sum_{j=1}^{N} j^{4} \dot{T}_{j, N}^{2}+C_{0} \sum_{j=1}^{N} j^{6} T_{j, N}^{2} \\
& +3 C_{1} \int_{0}^{\pi}\left(\sum_{j=1}^{N} j T_{j, N} \cos j x\right)^{2}\left(\sum_{j=1}^{N} j^{3} T_{j . N} \cos j x\right)^{2} d x \\
= & (2 / \pi)\left\langle w_{x x t}^{(N)}, w_{x x t}^{(N)}\right\rangle+\left(2 C_{0} / \pi\right)\left\langle w_{x x x}^{(N)}, w_{x x x}^{(N)}\right\rangle+3 C_{1}\left\langle w_{x}^{(N)^{2}}, w_{x x x}^{(N)^{2}}\right\rangle^{2}
\end{aligned}
$$


The object now is to estimate the right side of (2.10) in terms of $E_{N}$. For this purpose it is convenient to note that, since $w^{(N)}(0, t)=w^{(N)}(\pi, t)=$ 0 , Rolle's theorem implies the existence of a point $\zeta=\zeta(t)$ such that $w_{x}^{(N)}(\zeta, t)=0$. Therefore

(2.12) $\left|w_{x}^{(N)}\right| \leqq\left|\int_{\zeta}^{x} w_{x x}^{(N)} d x\right| \leqq \int_{0}^{\pi}\left|w_{x x}^{(N)}\right| d x \leqq\left(\pi \int_{0}^{\pi} w_{x x}^{(N)^{2}} d x\right)^{1 / 2}$.

In addition $w_{x x}^{(N)}(0, t)=0$ so that

$$
\left|w_{x x}^{(N)}\right|=\left|\int_{0}^{x} w_{x x x}^{(N)} d x\right| \leqq \int_{0}^{\pi}\left|w_{x x x}^{(N)}\right| d x \leqq\left(\pi \int_{0}^{\pi} w_{x x x}^{(N)^{2}} d x\right)^{1 / 2}
$$

Similarly it is easily shown that

$$
\left|w_{x t}^{(N)}\right| \leqq .\left(\pi \int_{0}^{\pi} w_{x x t}^{(N)^{2}} d x\right)^{1 / 2}
$$

The inequalities (2.12), (2.13), and (2.14) yield pointwise estimates on $w_{x}^{(N)}, w_{x x}^{(N)}$, and $w_{x t}^{(N)}$ in terms of $E_{N}$. Thus

$$
\begin{aligned}
& \left|w_{x}^{(N)}\right| \leqq\left(\pi^{2} /\left(2 C_{0}\right)^{1 / 2}\right) E_{N}^{1 / 2} \\
& \left|w_{x x}^{(N)}\right| \leqq\left(\pi /\left(2 C_{0}\right)^{1 / 2}\right) E_{N}^{1 / 2} \\
& \left|w_{x t}^{(N)}\right| \leqq\left(\pi / 2^{1 / 2}\right) E_{N}^{1 / 2} .
\end{aligned}
$$

The first term on the right of (2.10) can be estimated by

$$
\left|\left\langle w_{x}^{(N)} w_{x t}^{(N)}, w_{x x x}^{(N)^{2}}\right\rangle\right| \leqq \frac{\pi^{3}}{2 C_{0}^{1 / 2}} E_{N} \int_{0}^{\pi} w_{x x x}^{(\mathrm{N}))^{2}} d x \leqq \frac{\pi^{4}}{4 C_{0}^{3 / 2}} E_{N}^{2} .
$$

For the second term on the right of (2.10) integrate once by parts so that

$$
\begin{aligned}
\left|\left\langle w_{x}^{(N)} w_{x x}^{(N)^{2}}, w_{x x x t}^{(N)}\right\rangle\right|= & \left|\left\langle w_{x x}^{(N)^{3}}, w_{x x t}^{(N)}\right\rangle+2\left\langle w_{x}^{(N)} w_{x x}^{(N)} w_{x x x}^{(N)}, w_{x x t}^{(N)}\right\rangle\right| \\
\leqq & \frac{\pi^{3}}{\left(2 C_{0}\right)^{3 / 2}} E_{N}^{3 / 2}\left(\pi \int_{0}^{\pi} w_{x x t}^{(N)^{2}} d x\right)^{1 / 2} \\
& +\frac{\pi^{3}}{C_{0}} E_{N}\left(\int_{0}^{\pi} w_{x x x}^{(N)^{2}} d x \int_{0}^{\pi} w_{x x t}^{(N)^{2}} d x\right)^{1 / 2} \\
\leqq & \left(\pi^{4} / 4 C_{0}^{3 / 2}\right) E_{N}^{2}+\left(\pi^{4} / 2 C_{0}^{3 / 2}\right) E_{N}^{2} .
\end{aligned}
$$

In view of the inequalities (2.18) and (2.19) the identity (2.10) can be replaced by

$$
d E_{N} / d t \leqq\left(21 \pi^{4} / 2 C_{0}^{3 / 2}\right) E_{N}^{2}
$$

The inequality (2.20) yields the desired estimate on $E_{N}$. 
Lemma 1. Assume

$$
\begin{aligned}
\lim _{N \rightarrow \infty} E_{N}(0)= & e=\sum_{j=1}^{\infty} j^{4} \beta_{j}^{2}+C_{0} \sum_{j=1}^{\infty} j^{6} \alpha_{j}^{2} \\
& +3 C_{1} \int_{0}^{\pi}\left(\sum_{j=1}^{\infty} j \alpha_{j} \cos j x\right)^{2}\left(\sum_{j=1}^{\infty} j^{3} \alpha_{j} \cos j x\right)^{2} d x<\infty,
\end{aligned}
$$

i.e. assume $E_{N}(0)$ converges as $N \rightarrow \infty$. Then $E_{N}(t)$ is uniformly bounded independent of $N$ on any closed subinterval $0 \leqq t \leqq t^{*}<t_{c}$ where

$$
t_{c}=2 C_{0}^{3 / 2} / 21 \pi^{4} e .
$$

Proof. The inequality (2.20) implies that

$$
E_{N}(t) \leqq \frac{E_{N}(0)}{1-\left(21 \pi^{4} / 2 C_{0}^{3 / 2}\right) E_{N}(0) t}
$$

if

$$
0 \leqq t<2 C_{0}^{3 / 2} / 21 \pi^{4} E_{N}(0) .
$$

The lemma follows after taking the limit as $N \rightarrow \infty$. Q.E.D.

The bound on $E_{N}$ furnished by Lemma 1 is the key feature in proving the functions $T_{j}$, i.e. the limits of the subsequence $T_{j . N_{i}}$, are solutions of (1.1). In fact this result is a consequence of the following two lemmas:

Lemma 2. If $e<\infty(c f .(2.21))$ the infinite series

$$
\sum_{j=1}^{\infty} j^{6} T_{j}^{2}
$$

converges in the interval $0 \leqq t \leqq t^{*}<t_{c}$.

LEMMA 3. If $e<\infty$ the functions $w^{\left(N_{i}\right)}$ and $w_{x}^{\left(N_{i}\right)}$ converge to $w$ and $w_{x}$,

$$
w=\sum_{j=1}^{\infty} T_{j} \sin j x,
$$

as $N_{i} \rightarrow \infty$ for $t$ in the interval $0 \leqq t \leqq t^{*}<t_{c}$.

Proof of Lemma 2. The sequence of functions

$$
S_{n}=\sum_{j=1}^{n} j^{6} T_{j}^{2}
$$

is monotone increasing i.e. $S_{n+1} \geqq S_{n}$. Thus the convergence of $S_{n}$ as $n \rightarrow \infty$ will follow if it can be shown that $S_{n}$ is bounded independent of $n$. 
However, this is a consequence of Lemma 1 since

$$
\begin{aligned}
S_{n} & \leqq\left|S_{n}-\sum_{j=1}^{n} j^{6} T_{j, N_{i}}^{2}\right|+\sum_{j=1}^{N_{i}} j^{6} T_{j, N_{i}}^{2} \\
& \leqq\left|S_{n}-\sum_{j=1}^{n} j^{6} T_{j, N_{i}}^{2}\right|+\frac{1}{C_{0}} E_{N_{i}}(t) .
\end{aligned}
$$

The function $E_{N_{i}}$ is bounded independent of $N_{i}$ in the interval $0 \leqq t \leqq t^{*}$. Therefore the lemma follows upon taking the limit as $N_{i} \rightarrow \infty$. Q.E.D.

ProOf OF LemMa 3. The fact that $w$ and $w_{x}$ converge on the interval $0 \leqq t \leqq t^{*}$ follows from the Schwarz inequality and Lemma 1 . In order to show that $w_{x}^{\left(N_{i}\right)} \rightarrow w_{x}$ note that Lemma 1 implies that $T_{j}^{2} \leqq M / j^{6}$ and $T_{j, N_{i}}^{2} \leqq$ $M / j^{6}$ where $M$ is a constant determined by the bound on $E_{N}$ in the interval $0 \leqq t \leqq t^{*}$. Therefore

$$
\begin{aligned}
\left|w_{x}-w_{x}^{\left(N_{i}\right)}\right| \leqq & \left|\sum_{j=1}^{n} j\left(T_{j}-T_{j, N_{i}}\right) \cos j x\right| \\
& +\sum_{j=n+1}^{\infty} j\left|T_{j}\right|+\sum_{j=n+1}^{N_{i}} j\left|T_{j, N_{i}}\right| \\
& +\sum_{j=1}^{n} j\left|T_{j}-T_{j, N_{i}}\right|+2 M^{1 / 2} \sum_{j=n+1}^{\infty} \frac{1}{j^{2}} .
\end{aligned}
$$

We can make the right side of (2.29) small by first choosing $n$ and then choosing $N_{i}$. The proof that $w^{\left(N_{i}\right)} \rightarrow w$ is done in a similar manner. Q.E.D.

THEOREM 1. The functions $T_{j}$ are a solution of (1.1) satisfying the initial conditions (1.2) and auxiliary condition (1.3) in the interval $0 \leqq t \leqq t^{*}<t_{c}$ if $e<\infty$.

Proof. The functions $T_{j, N_{i}}$ satisfy the Volterra integral equation

$$
\begin{aligned}
T_{j, N_{i}} & =\alpha_{j}+\beta_{j} t-\int_{0}^{t}(t-\tau)\left\{C_{0} j^{2} T_{j, N_{i}}+C_{1} j\left\langle w_{x}^{\left(N_{i}\right)^{3}}, \cos j x\right\rangle\right\} d \tau \\
& =\alpha_{j}+\beta_{j} t-G_{j} w_{x}^{\left(N_{i}\right)}
\end{aligned}
$$

for $j=1,2, \cdots, N_{i}$. The object is to show that the functions $T_{j}$ satisfy a similar equation. For this purpose write $\left(\|\cdot\|=\max _{0 \leqq t \leqq t^{*}}|\cdot|\right)$

$$
\begin{aligned}
\left|T_{j}-\alpha_{j}-\beta_{j} t+G_{j} w_{x}\right|= & \left|T_{j}-T_{j, N_{i}}-G_{j} w_{x}^{\left(N_{i}\right)}+G_{j} w_{x}\right| \\
\leqq & \left\|T_{j}-T_{j, N_{i}}\right\|+C_{0} j^{2} t^{*}\left\|T_{j}-T_{j . N_{i}}\right\| \\
& +C_{1} j t^{*}\left\|\left\langle w_{x}^{\left(N_{i}\right)^{3}}-w_{x}^{3}, \cos j x\right\rangle\right\| .
\end{aligned}
$$

The right side of (2.31) approaches zero as $N_{i} \rightarrow \infty$. Therefore $T_{j}$ is a 
solution of

$$
T_{j}=\alpha_{j}+\beta_{j} t-G_{j} w_{x} .
$$

The theorem follows on differentiation of (2.32). Q.E.D.

The essential feature in the proof of Theorem 1 is of course the bound obtained in Lemma 1. The interval over which this bound is obtained, i.e. $0 \leqq t \leqq t^{*}<t_{c}$, can be increased slightly by a more careful estimate of the right-hand side of (2.10). However, since no real qualitative change in the results are obtained the details are omitted. It is worth noting, however, that although the interval of existence $0 \leqq t \leqq t^{*}<t_{c}$ is finite it may be large indeed. In fact as the initial data gets small $(e \rightarrow 0)$ or as $C_{0} \rightarrow \infty$ the interval of existence also becomes infinite, i.e. $t_{c} \rightarrow \infty$.

The most serious difficulty in the above development is that, because of the use of the Arzela-Ascoli lemma, the existence proof is not truly constructive. Thus it is conceivable that there exists a second sequence of functions, say $\left\{T_{j, N_{l}}\right\}$, converging to limit functions which differ from $T_{j}$ and hence define a different solution to (1.1). Consequently a uniqueness theorem plays an important role in the proof of existence. In fact it can be shown that (1.1) has at most one solution satisfying the initial data (1.2) and auxiliary condition (1.3). However, since the proof of this fact is relatively straightforward (cf. [4]) it will not be included here.

In $\S 1$ of this paper it was indicated that the proof of existence of solutions to (1.1) satisfying the conditions (1.2) and (1.3) is sufficient to prove the existence of a classical solution to (1.4) satisfying the conditions (1.5) and (1.6). To see why this is true assume that the periodic continuation of $f(x)$ and $g(x)$ is sufficiently differentiable to guarantee that

$$
\begin{aligned}
& \alpha_{j}=\frac{2}{\pi} \int_{0}^{\pi} f(x) \sin j x d x, \\
& \beta_{j}=\frac{2}{\pi} \int_{0}^{\pi} g(x) \sin j x d x
\end{aligned}
$$

satisfies the condition (2.21). In this case Theorem 1 guarantees the existence of a solution to (1.1) satisfying the conditions (1.2) and (1.3) in the interval $0 \leqq t \leqq t^{*}<t_{c}$. It follows from (1.3) that the function

$$
w(x, t)=\sum_{j=1}^{\infty} T_{j}(t) \sin j x
$$

is twice continuously differentiable with respect to $x$ and $t$. Thus if the nonlinear operator defined by (1.4) is applied to (2.34) the result is a function, say $C(x, t)$, which is certainly continuous. The object is to show that it zero. However, this follows upon noting that $\langle C(x, t), \sin j x\rangle=0$ for all 
$j$ since this is just the infinite system (1.1). Therefore $C(x, t)$ is a continuous function which is orthogonal to every member of a complete orthogonal set. The conclusion is that $C(x, t) \equiv 0$ and hence $(2.34)$ is the desired solution.

\section{REFERENCES}

1. R. Narasimha, Non-linear vibrations of an elastic string, J. Sound. Vib. 8 (1968), 134-146.

2. S. Woinowsky-Krieger, The effect of axial force on the vibration of hinged bars, $\mathrm{J}$. Appl. Mech. 17 (1950), 35-36. MR 11, 558.

3. R. W. Dickey, Free vibrations and dynamic buckling of the extensible beam, J. Math. Anal. Appl. 29 (1970), 443-454. MR 40 \#6831.

4. - Infinite systems of nonlinear oscillation equations related to the string, Proc. Amer. Math. Soc. 23 (1969), 459-468. MR 40 \#458.

5. J. M. Ball, Initial boundary value problems for an extensible beam, J. Math. Anal. Appl. (to appear).

6. E. A. Coddington and N. Levinson, Theory of ordinary differential equations, McGraw-Hill, New York, 1955. MR 16, 1022.

7. N. J. Zabusky, Exact solution for the vibrations of a nonlinear continuous model string, J. Mathematical Phys. 3 (1962), 1028-1039. MR 26 \#4067.

8. P. D. Lax, Development of singularities of solutions of nonlinear hyperbolic partial differential equations, J. Mathematical Phys. 5 (1964), 611-613. MR 29 \#2532.

9. R. C. MacCamy and V. J. Mizel, Existence and nonexistence in the large of solutions of quasilinear wave equations, Arch. Rational Mech. Anal. 25 (1967), 299-320. MR 35 \#7000.

Department of Mathematics, University of Wisconsin, Madison, Wisconsin 53706 